\title{
Idea Management Systems as a Tool for Goals' Setting \& Achieving and Decision Making: Global Research Results
}

\author{
Elina Mikelsone ${ }^{1 ; 2, *}$, Tatjana Volkova $^{2}$, Aivars Spilbergs ${ }^{2}$, Elita Liela $^{2}$ \\ ${ }^{1}$ Idea Innovation Institute, Ltd. \\ ${ }^{2}$ BA School of Business and Finance, the Faculty of Business Administration, 161, Kr.Valdemara \\ Str., LV-1013, Riga, Latvia
}

\begin{abstract}
Research background: the authors have explored that there are different idea management system (IMS) application types that could be used both locally and globally for diverse reasons and expected outcomes. There is ongoing research on how IMS could be applied for manageable idea management process. But there is a question - how do these IMS types help to set and achieve goals, and improve decision making?

Purpose of the article: The article aims to clarify how an external and mixed web-based IMS could be used during COVID19 time for distance idea generation sessions, as well as, to solve complex issues such as decision making, goals' setting and reaching them based on different idea generation sources and critical reflection on those ideas of evaluators.

Methods: Literature review (data collection: systematic data collection from scientific data bases; data analysis: content analysis). The survey of $n>400$ enterprises with web-based IMS experience globally (data collection: a survey; data analysis: statistics).

Findings \& Value added: this paper explores how different types of webbased IMS could be applied as a tool and support system for decision making processes in general, decisions towards goal setting and its outreach. The research results provide also a practical contribution - it could help to choose the most appropriate IMS application type to reach estimated goals and to empower decision making.
\end{abstract}

Keywords: Idea Management Systems; Web-based Idea Management Systems; Decision Making, Goal Setting

JEL Classification: L86; D89; D79; $M 15$

\footnotetext{
* Corresponding author: mikelsone.elina@gmail.com
} 


\section{Introduction}

Ideas turned in different types of innovations are crucial aspects of creativity economy - a model that colides creativity, knowledge, and innovation economies (Dublina et al., 2012), innovation-driven economy (Wonglimpiyarat \& Khaemasunun, 2017), knowledge economy (Rim et al., 2019), circular economy (Khan \& Haleem, 2021) and other types of economies' development nowadays. Ideas could come not only from organizations but also behind the walls of the company, for example, with idea crowdsourcing contests (Mazzola et al., 2020; Steils \& Hanine, 2019). Idea crowdsourcing contests help to organize the process how to materialize idea creator's creativity in ideas that the organization needs (Natalicchio et al., 2017). There is a common agreement among researchers that open innovation is a driving force for improving organizations performance (Vincenzi \&Cunha (2021). There are many approaches how to materialize creativity in innovations. One of such tools is idea management system.

Idea management systems (IMS) provide systematic and manageable idea management (IM) process - idea generation and evaluation. Idea contests, crowdsourcing, open innovation platforms have helped many organizations all around the world. The common element in all these systems is IM process.

The authors have explored, that there are different idea management system (IMS) application types that could be used both locally and globally: an active and a passive one, an internal and an external one, as well as a mixed one. An active IMS helps to collect ideas in idea contests, but a passive IMS just offers opportunity to submit all ideas at the same time. An internal IMS collects ideas from employees, an external IMS based on crowdsourcing does it from crowds or other external sources, a mixed IMS provides both approaches.

There is no common understanding among researchers how do these IMS types help to set and achieve goals, and improve decision making.

The article aims to clarify how an external and a mixed web-based IMS could be used during COVID19 time for distance idea generation sessions, as well as to solve complex issues based on different idea generation sources and evaluators, thus empowering decision making, goal setting and reaching them. The following hypothesis statements have been tested:

1. The application of any type of IMS has helped in goals' setting;

2. The application of any type of IMS has improved decision making;

3. The application of any type of IMS has helped to achieve the set goals.

Research methods: Literature review (data collection: systematic data collection from scientific data bases; data analysis: content analysis). Main scientific data bases: Scopus and Web of Science. Mainly scientific articles and proceedings were applied. The survey of $\mathrm{n}>400$ enterprises with web-based IMS experience (data collection: a survey; data analysis: statistics).

This paper explores how different types of web-based IMS could be applied as a support system for decision making processes in general, decisions towards goal setting and its outreach.

\section{Methods}

Researchers conducted the survey of IMS users. The survey was distributed through 107 IMS providers and they sent it to their clients. The survey of $n>400$ enterprises with web-based IMS experience (data collection: a survey; data analysis: statistics). It should be noted that there are approximately 120000 web-based IMS users all over the world. 
Hypothesis testing approach is based on theoretical guidelines (Moore et al., 2016). For hypotheses testing the authors will use the proportion $\left(P_{\mathrm{i}}\right)$ of respondents who believe that the application of a particular type of IMS has improved a specific area:

$$
P_{i}=\frac{k_{i}}{n_{i}}
$$

where $k_{\mathrm{i}}$ - the number of respondents who believe that the application of IMS has improved a specific area;

$n_{\mathrm{i}}-$ the number of respondents applying IMS.

For the tests against hypothesized proportion $P_{0}=0.5$ on a total sample level null and an alternative hypothesis is defined as follows:

$$
H_{0}: P_{\mathrm{i}}-P_{0} \leq 0 \text { and } H_{\mathrm{A}}: P_{\mathrm{i}}-P_{0}>0
$$

As sample is a simple random and independent, and $k>120 \&(n-k)>40$, the difference of two proportions follows an approximate normal distribution and the test statistic is calculated as follows:

$$
Z=\frac{P_{i}-P_{0}}{\sqrt{P_{0} *\left(1-P_{0}\right) / n}}
$$

The decision rule is to reject $H_{0}$ in favour of $H_{\mathrm{A}}$, if $z$-statistic $\left(z_{\text {stat }}\right)$ is larger than $z$-critical $\left(z_{\alpha}\right)$ values of $(\alpha=0.05)$ :

$$
z_{\text {stat }}>z_{\alpha}
$$

For the tests at a particular IMS type level null and an alternative hypothesis is defined as follows:

$$
H_{0}: P_{\mathrm{i}}-P_{\mathrm{j}} \leq 0 \text { and } H_{\mathrm{A}}: P_{\mathrm{i}}-P_{\mathrm{j}}>0
$$

As samples are simple random and independent, and $k>80 \&(n-k)>30$, the difference of two proportions follows an approximate normal distribution and the test statistic is calculated as follows:

$$
Z=\frac{P_{i}-P_{j}}{\sqrt{P_{c} *\left(1-P_{c}\right) *\left(\frac{1}{n_{i}}+\frac{1}{n_{j}}\right)}}
$$

where the pooled proportion $\left(P_{c}\right)$ is calculated as follows:

The decision rule is defined above (4).

$$
P_{C}=\frac{k_{i}+k_{j}}{n_{i}+n_{M j}}
$$

\section{Results}

\subsection{Findings of Hypotheses}

Based on the survey results, variable values where fixed and together with the test statistics and corresponding $p$-values summarized in the following Table 1.

Table 1. Variable values, test statistics and corresponding $p$-values.

\begin{tabular}{|c|r|r|r|r|c|}
\hline \# hypothesis & \multicolumn{1}{|c|}{$\boldsymbol{n}$} & \multicolumn{1}{c|}{$\boldsymbol{k}$} & \multicolumn{1}{c|}{$\boldsymbol{P}_{\boldsymbol{0}}$} & \multicolumn{1}{c|}{$\boldsymbol{z}_{\text {stat }}$} & $\boldsymbol{p}$-value \\
\hline 1 & 185 & 121 & 0.5 & 4.1907 & $<0.0001$ \\
\hline 2 & 186 & 144 & 0.5 & 7.4790 & $<0.0001$ \\
\hline 3 & 205 & 164 & 0.5 & 8.1609 & $<0.0001$ \\
\hline
\end{tabular}

Source: created by the authors

As calculated $z$-statistics is larger than a critical value for all hypotheses, the authors would reject the corresponding null hypotheses and conclude that sample data provide sufficient evidence that the application of any type of IMS has helped to set and achieve the goals, and improved decision making. Conclusions that the application of IMS has helped to 
set and achieve the goals, and improved decision making are supported also by very low pvalues $(<0,0001)$.

\subsection{Application of Different IMS Types and Goal Setting}

The following additional research questions as regards to the first hypothesis arose during the study:

a) the application of an active IMS type has helped to set the goals more than the application of a passive IMS type;

b) the application of an internal IMS type has helped to set the goals more than the application of an external IMS type;

c) the application of a mixed IMS type has helped to set the goals more than application of an active IMS type.

The results of the survey, which show the extent to which different types of IMS have helped to set the goals, are summarized in the following Figure 1.

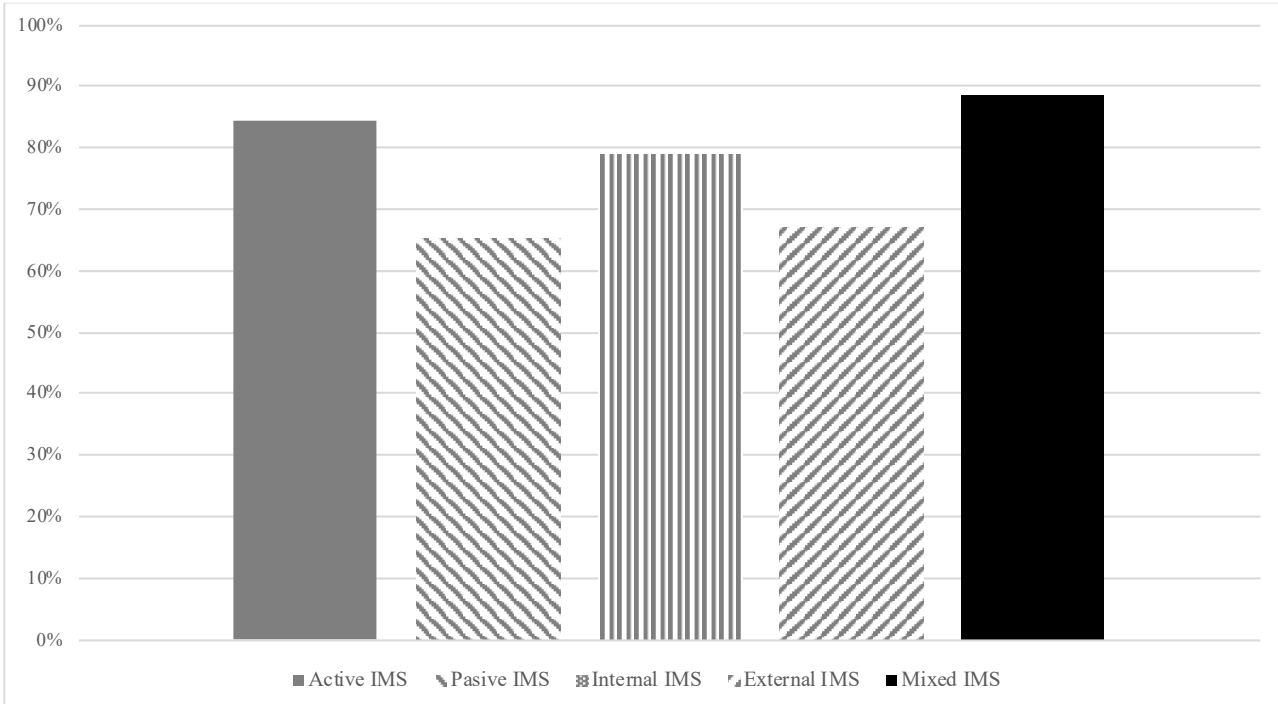

Figure 1. The proportion of respondents who believe that the application of a particular IMS type has helped to set the goals.

Source: created by the authors

Calculated proportions of respondents who believe that the applications of a particular IMS type has helped to set the goals, pooled proportions, as well as z-statistics and corresponding p-values are summarized in the following Table 2.

Table 2. Statistics for hypothesis testing about the setting of goals

\begin{tabular}{|l|l|l|l|l|l|l|}
\hline IMS type & $\boldsymbol{P}_{\boldsymbol{i}}$ & IMS type & $\boldsymbol{P}_{\boldsymbol{j}}$ & $\boldsymbol{P}_{\boldsymbol{c}}$ & $\boldsymbol{z}_{\text {stat }}$ & $\boldsymbol{p}$-value \\
\hline Active & 0.8454 & Passive & 0.6541 & 0.7876 & 5.3159 & $<0.0001$ \\
\hline Internal & 0.7899 & External & 0.6699 & 0.7342 & 2.8549 & 0.0022 \\
\hline Mixed & 0.8867 & Active & 0.8454 & 0.8609 & 1.5094 & 0.0657 \\
\hline
\end{tabular}

Source: created by the authors

As calculated z-statistics is larger than critical values for the first two comparable IMS pair types, the authors would reject the corresponding null hypothesis and conclude that the sample data provides a strong evidence to support an alternative hypothesis. Conclusions that 
the application of an active IMS has helped to set the goals more than the application of a passive IMS and the application of an internal IMS has helped to set the goals more than the application of an external IMS is supported also by a low $p$-value $(<0.0023)$.

As calculated z-statistic is not larger than critical values for the third comparable IMS types pairs, the authors cannot reject the corresponding null hypothesis and conclude that the sample data does not provide enough evidence to support an alternative hypothesis - the difference in the proportions of respondents who believe that the application of a mixed IMS has helped to set the goals more than the application of an active IMS is not statistically significant.

\subsection{Application of Different IMS Types and Decision-Making}

The following additional questions as regards to the second hypothesis arose during the study:

a) the application of an active IMS type has improved decision making more than the application of a passive IMS type;

b) the application of an internal IMS type has improved decision making more than the application of an external IMS type;

c) the application of a mixed IMS type has improved decision making more than the application of an active IMS type.

The results of the survey, which show the extent to which different types of IMS have improved decision making, are summarized in the following Figure 2.

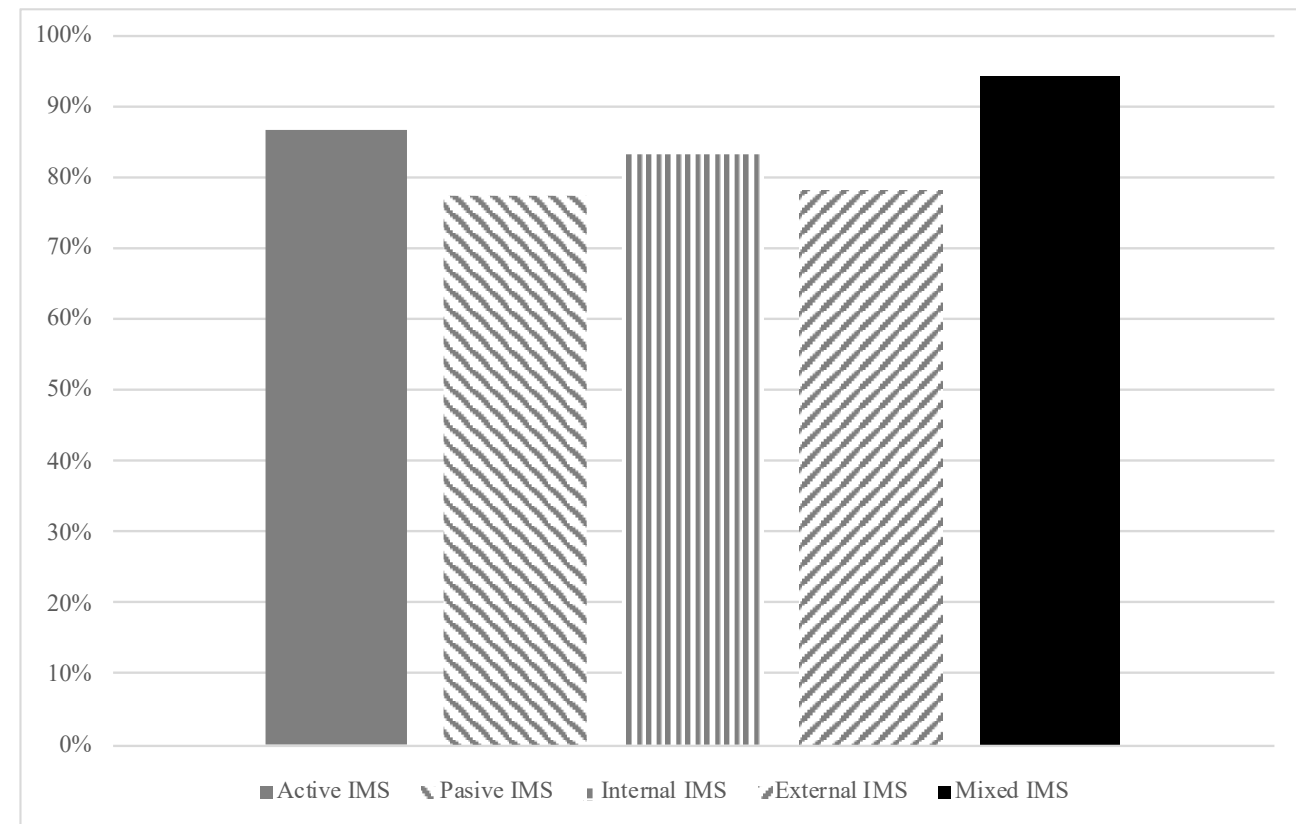

Figure 2. The proportion of respondents who believe that the application of a particular IMS type has improved decision making.

Source: created by the authors

Calculated proportions of respondents who believe that the applications of a particular IMS type has improved decision making, pooled proportions as well as $z$-statistics and corresponding $p$-values are summarized in the following Table 3. 
Table 3. Statistics for hypothesis testing about decision making

\begin{tabular}{|l|c|l|c|c|c|r|}
\hline IMS type & $\boldsymbol{P}_{\boldsymbol{i}}$ & IMS type & $\boldsymbol{P}_{\boldsymbol{j}}$ & $\boldsymbol{P}_{\boldsymbol{c}}$ & $\boldsymbol{z}_{\text {stat }}$ & $\boldsymbol{p}$-value \\
\hline Active & 0.8674 & Passive & 0.7742 & 0.8393 & 2.8931 & 0.002 \\
\hline Internal & 0.8326 & External & 0.7816 & 0.8090 & 1.3669 & 0.086 \\
\hline Mixed & 0.9419 & Active & 0.8674 & 0.8953 & 3.0872 & 0.001 \\
\hline
\end{tabular}

Source: created by the authors

As calculated z-statistics is larger than critical values for the first and the third comparable IMS pair types, the authors would reject the corresponding null hypothesis and conclude that the sample data provides a strong evidence to support an alternative hypothesis. Conclusions that the application of an active IMS has improved decision making more than the application of a passive IMS and the application of a mixed IMS has improved decision making more than the application of an active IMS is supported also by a low $p$-value $(<0.002)$.

As calculated z-statistic is not larger than critical values for the second comparable IMS pair types, the authors cannot reject the corresponding null hypothesis and conclude that the sample data does not provide enough evidence to support an alternative hypothesis - the difference in the proportions of respondents who believe that the application of an internal IMS type has improved decision making more than the application of an external IMS type is not statistically significant.

\subsection{Application of Different IMS Types and Goal Achievements}

The following additional research questions as regards to the second hypothesis arose during the study:

a) the application of an active IMS type has helped to achieve goals more than the application of a passive IMS type;

b) the application of an internal IMS type has helped to achieve goals more than the application of an external IMS type;

c) the application of mixed IMS type has helped to achieve goals more than the application of an active IMS type.

The results of the survey showing the extent to which different types of IMS have helped to achieve goals, are summarized in the following Figure 3. 


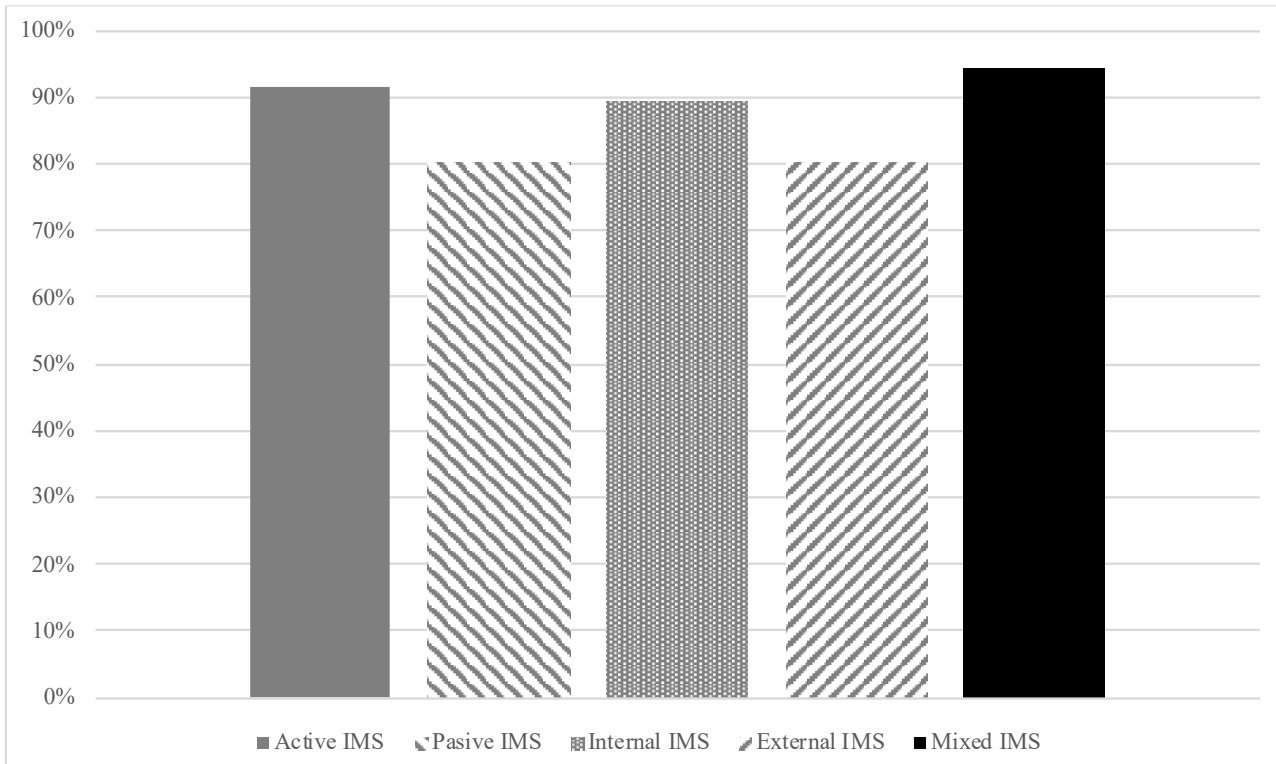

Figure 3. The proportion of respondents who believe that the application of a particular IMS type has helped to achieve goals

Source: created by the authors

Calculated proportions of respondents who believe that the applications of a particular IMS type has helped to achieve goals, pooled proportions, as well as z-statistics and corresponding p-values are summarized in the following Table 4.

Table 4. Statistics for hypothesis testing about goal achievement

\begin{tabular}{|l|l|l|l|l|l|l|}
\hline IMS type & $\boldsymbol{P}_{\boldsymbol{i}}$ & IMS type & $\boldsymbol{P}_{\boldsymbol{j}}$ & $\boldsymbol{P}_{\boldsymbol{c}}$ & $\boldsymbol{z}_{\text {stat }}$ & $\boldsymbol{p}$-value \\
\hline Active & 0.9138 & Passive & 0.8000 & 0.8795 & 3.9724 & $<0.0001$ \\
\hline Internal & 0.8954 & External & 0.8000 & 0.8514 & 2.8170 & 0.0024 \\
\hline Mixed & 0.9419 & Active & 0.9138 & 0.9243 & 1.3488 & 0.0887 \\
\hline
\end{tabular}

Source: created by the authors

As calculated z-statistics is larger than critical values for the first two comparable IMS pair types, the authors would reject the corresponding null hypothesis and conclude that the sample data provides a strong evidence to support an alternative hypothesis. Conclusions that the application of an active IMS has helped to achieve goals more than the application of a passive IMS and the application of an internal IMS has helped to achieve goals more than the application of an external IMS is supported also by a low $p$-value $(<0.0025)$.

As calculated z-statistic is not larger than critical values for the third comparable IMS pair types, the authors cannot reject the corresponding null hypothesis and conclude that the sample data does not provide enough evidence to support an alternative hypothesis - the difference in the proportions of respondents who believe that the application of a mixed IMS has helped to helped to achieve goals more than the application of an active IMS is not statistically significant. 


\section{Discussion}

IMS, goals setting and decision making, achieving the goals - all these points involve humans - so they are influenced by social aspects. Altay et al. (2020) propose very important aspect in IM - idea appropriation. This is one of the social aspects that is very important in IMS context that should be researched in detail- how different IMS types impact it and how idea appropriation impacts goal setting, decision making and achieving of goals as the final outcome.

In this paper the goal setting is simplified, but in the future research it could be researched in detail, because other research findings suggest that the goal setting is a complex process. (Eckhoff \& Weiss, 2020). In this case the focus was an overall goal setting at the organizational level, but it could be researched also at a group and individual levels. The role of IMS in the goal setting could be researched from a creative organizations' perspective where often the outcomes are difficult to foresee. Different approaches are needed for setting goals and making decisions based on the time perspective: a short term, a medium term and a long term. With the goal setting are closely related such terms as the group 1: confidence, commitment, motivation, and knowledge; the group 2: specific, challenging, measurable, and relevant within a specified amount of time (Eckhoff \& Weiss, 2020). The first elements could be researched as enablers for IM, but the group 2 as potential outputs for the goal setting - how well defined are they during IM. The goal setting element gives also the future research direction - how the group 2 elements could be used to set the goals for IM itself and how they influence the final results of IM. Additionally, there could be researched IMS type application relations with different goal setting styles as enablers, but a goal commitment (van Lill et al., 2020) as an output for different IMS application types. The competence of IMS is the necessary prerequisite for a successful IM and its application. Therefore, the result of IMS application in decision making could be moderated based on the competence of idea management team. For different expected outcomes different idea management system types are more appropriate than another ones, e.g. for achieving goals regarding a company internationalization or a new product development.

Achieving goals is also researched as a general element, but in the future different goals and their settings and achievement could be researched, for example, focusing on specific goals, e.g. achieving sustainable development goals (Malesevic \& Kosor, 2020), achieving organizational goals (Bushi, 2021) etc.

A decision making element in this research also is not divided into stages, but it should, other researchers show a great effort exploring decision-making styles and decision-making stages (Virdi et al., 2020). An interesting element for the further discussions is different decision-making techniques (Tahiri et al., 2021), as well as, the impact on decision making of an organizational culture, etc.

In 2020 study the authors (Mikelsone et al., 2020) examined how different IMS application types impact idea quantity- it could be researched if the idea quantity was mediator in these relations between IMS application type - goal setting, decision making and goals' achievement.

\section{Conclusions}

The paper describes how different types of web-based IMS could be applied as a support system for decision making processes in general, decisions towards the goal setting and its outreach. The main results of the research could be summarized as follows: (1) the application of an active IMS has helped to set the goals more than the application of a passive IMS and the application of an internal IMS has helped to set the goals more than the application of an external IMS; (2) the application of an active IMS has improved decision 
making more than the application of a passive IMS and the application of a mixed IMS has improved decision making more than the application of an active IMS; (3) the application of an active IMS has helped to achieve goals more than the application of a passive IMS and the application of an internal IMS has helped to achieve goals more than the application of an external IMS.

The research results provide also a practical contribution - it could help to choose the most appropriate IMS application type to reach estimated goals and to empower decision making.

The future research directions based on the authors opinion could be related to: (1) the research on IM business models and their impact on these results; (2) the research on how IMS application types impact other outputs (for example, creativity, a product, a process, marketing and organizational innovations, a human resource performance, etc.); (3) the research of an impact of different mediators in these relationships (for example, rewards for idea creators, the duration of tasks, the type of tasks, engagement, etc.).

\section{Acknowledgements}

This work has been supported by the European Regional Development Fund within the Activity 1.1.1.2 "Postdoctoral Research Aid" of the Specific Aid Objective 1.1.1 "To increase the research and innovative capacity of scientific institutions of Latvia and the ability to attract external financing, investing in human resources and infrastructure" of the Operational Programme "Growth and Employment" (N-1.1.1.2/VIAA/4/20/670).

\section{References}

1. Altay, S., Majima, Y., \& Mercier, H. (2020). It's my idea! Reputation management and idea appropriation. Evolution and Human Behavior, 41(3), 235-243.

2. Bushi, F. (2021). An Overview of Motivation Theories: The Impact of Employee Motivation on Achieving Organizational Goals. Quality-Access to Success, 22(183), 812.

3. Dubina, I. N., Carayannis, E. G., \& Campbell, D. F. J. (2012). Creativity Economy and a Crisis of the Economy? Coevolution of Knowledge, Innovation, and Creativity, and of the Knowledge Economy and Knowledge Society. Journal of the Knowledge Economy, $3(1), 1-24$.

4. Eckhoff, D. O., \& Weiss, J. (2020). Goal setting: A concept analysis. Nursing Forum, 55(2), 275-281.

5. Khan, S., \& Haleem, A. (2021). Investigation of circular economy practices in the context of emerging economies: a CoCoSo approach. International Journal of Sustainable Engineering, 14(3), 357-367.

6. Malesevic Perovic, L., \& Kosor, M. M (2020). The Efficiency of Universities in Achieving Sustainable Development Goals. Amfiteatru Economic, 22(54), 516-532.

7. Mazzola, E., Piazza, M., Acur, N., \& Perrone, G. (2020). Treating the crowd fairly: Increasing the solvers' self-selection in idea innovation contests. Industrial Marketing Management, 91, 16-29.

8. Mikelsone, E., Spilbergs, A., Volkova, T., Liela, E. \& Frisfelds, J. (2020). Idea Management System Application Type Impact on Idea Quantity. European Integration Studies, 14, 192-20.

9. Moore, D., McCabe, G., Alwan, L., \& Craig, B. (2016). The practice of statistics for business and economics. 4th ed. W. H. Freeman \& Co. 
10. Natalicchio, A., Messeni Petruzzelli, A., \& Garavelli, A. C. (2017). Innovation problems and search for solutions in crowdsourcing platforms - A simulation approach. Technovation, 64-65, 28-42.

11. Rim, G. N., Kim, G. S., Hwang, S. H. \& Ko, U. D. (2019). Some Problems in Statistically Assessing the Level of Knowledge Economy. Journal of the Knowledge Economy, 10(3), 974-996.

12. Steils, N., \& Hanine, S. (2019). Recruiting valuable participants in online IDEA generation: The role of brief instructions. Journal of Business Research, 96, 14-25.

13. Tahiri, A., Kovaci, I., Bushi, F., \& Meha, A. (2021). Decision-Making and the Applying of Decision-Making Techniques in SMEs in Kosovo. Quality-Access to Success, 22(180), 64-67.

14. van Lill, X., Roodt, G., \& de Bruin, G. P. (2020). The relationship between managers' goal-setting styles and subordinates' goal commitment. South African Journal of Economic and Management Sciences, 23(1), a3601.

15. Vincenzi, T. B. D., \& da Cunha, J. C. (2021). Open innovation and performance in the service sector. Innovation \& Management Review, Early acces.

16. Virdi, P., Kalro, Aa. D., \& Sharma, D. (2020). Online decision aids: the role of decisionmaking styles and decision-making stages. International Journal of Retail \& Distribution Management, 48(6), 555-574.

17. Wonglimpiyarat J., \& Khaemasunun P. (2017). Strategies of remodelling China towards an innovation-driven economy. International Journal of Business Innovation and Research, 12(2), 175-188. 